\title{
Crushed Jaw Fracture in a Patient with Cleidocranial Dysplasia
}

\section{ISSN: 2578-0379}

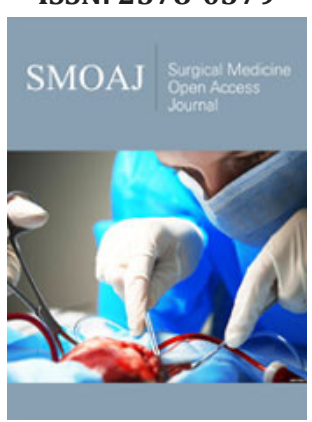

*Corresponding author: Bruno Da Silva Gaspar, School of Dentistry, Brazil

Submission: 制April 04, 2019

Published: 此April 12, 2019

Volume 2 - Issue 4

How to cite this article: Bruno D S G, John K S D C, Rafael L A, Bruno F A S. Crushed Jaw Fracture in a Patient with Cleidocranial Dysplasia. Surg Med Open Acc J.2(4). SMOAJ.000542.2019. DOI: 10.31031/SMOAJ.2019.02.000542.

Copyright@ Bruno Da Silva Gaspar, This article is distributed under the terms of the Creative Commons Attribution 4.0 International License, which permits unrestricted use and redistribution provided that the original author and source are credited.

\author{
Bruno Da Silva Gaspar ${ }^{*}$, John Kleber Sales De Castroํ, Rafael Linard Avelar ${ }^{2}$ \\ and Bruno Frota Amora Silva ${ }^{2}$ \\ ${ }^{1}$ School of Dentistry, Brazil \\ ${ }^{2}$ Division of Oral and Maxillofacial Surgery, School of Dentistry, Brazil
}

\begin{abstract}
Cleidocranial Dysplasia is a rare congenital syndrome that presents a wide range of expressions. This condition can affect the bones of both the endochondral and intramembranous origins, resulting in generalized skeletal disorders. The skeletal manifestations of this syndrome tend to cause important changes in the skull, spine and teeth of affected individuals. This paper reports a rare case of a 45-year-old patient with Cleidocranial Dysplasia with a fractured mandibular angle after a domestic accident, which was attended at Waldemar Alcantara General Hospital (HGWA).
\end{abstract}

Keywords: Cleidocranial dysostosis; Mandibula injuries; Mutation; Autosomal dominant

\section{Introduction}

Cleidocranial Dysplasia, also known as Marie Sainton's disease or mutational dysostosis, is a rare autosomal dominant congenital bone disease with a wide range of expressions. The estimated prevalence is 1: 1,000,000, which can affect both sexes and all races [1,2].

Its main characteristics is the hypoplasia or aplasia of the clavicles, resulting in a greater range of movement of the shoulders. Patients suffering from this disease usually have short stature, having a skull disproportionate to their body. They present abnormal dentition, with defects in the eruption of deciduous teeth, delayed eruption of the definitive dentition and presence of supernumerary teeth $[1,3,4]$.

This article reports a case of a 45-year-old domestic accident victim with a broken mandibular angle fracture who was diagnosed with Cleidocranial Dysplasia only after trauma. A detailed clinical examination was done to collect data about the disease to perform new treatments in these patients.

\section{Case Report}

A 45-year-old man attended the outpatient clinic of the Oral and Maxillofacial Surgery Service of General Waldemar Alcantara Hospital reporting that he suffered a fall with facial trauma on the right side of the jaw. The patient complained of pain in the region of the trauma and reported much discomfort in any mandibular function.

Physical examination showed a man of short stature, with facial features of ocular hypertelorism, hypoplasia of the middle face and with relative mandibular prognathism. Intraoral oral examination revealed maxillary constriction, probably associated with the cleft palate underlying the mucosa, and several dental agenesis may be observed. Edema was observed in the right submandibular region, associated with blunt cut wounds in the dorsal region of the tongue. The patient denied any emetic episode or loss of consciousness.

Several skeletal changes were observed, such as clavicular deformity, which was easily observed by the high mobility of the shoulders. His hands were small and with short 
phalanges. It was also possible to observe a strange pattern of bone apposition in the skull, in the parietal and occipital region, and the clinical diagnosis of Cleidocranial Dysplasia was then proposed.

Radiographs of the patient's face, computed tomography (CT) and preoperative examinations were requested to perform the surgical procedure. A CT fracture of the mandibular angle was associated with the presence of three impacted teeth, where one of these teeth crossed the fractured region. In the 3D reconstruction, several Wormian bones were observed in the occipital, frontal and parietal regions.

The patient underwent surgery under general anesthesia, with nasotracheal intubation. The posterior region of the right side of the mandible was accessed via the submandibular way giving access to the fracture area. The displaced bone fragments were large and after removal of the bone fragment in the highest fracture region, it was possible to observe the presence of an unerupted tooth as well as the presence of the inferior alveolar nerve.

The bone segments were repositioned and a fixation with two titanium plates of the 2.0 system (region of the mandibular base) was performed and two screws of the 2.0 system were used to reattach the fractured clusters between the buccal and lingual sides of the fracture. After this simplification of the fracture was carried out the placement of a plate of the system of lock of $2,4 \mathrm{~mm}$ like form of type of fixation of the support of load. No communication with the intraoral mucosa was observed and the surgical wounds were sutured.

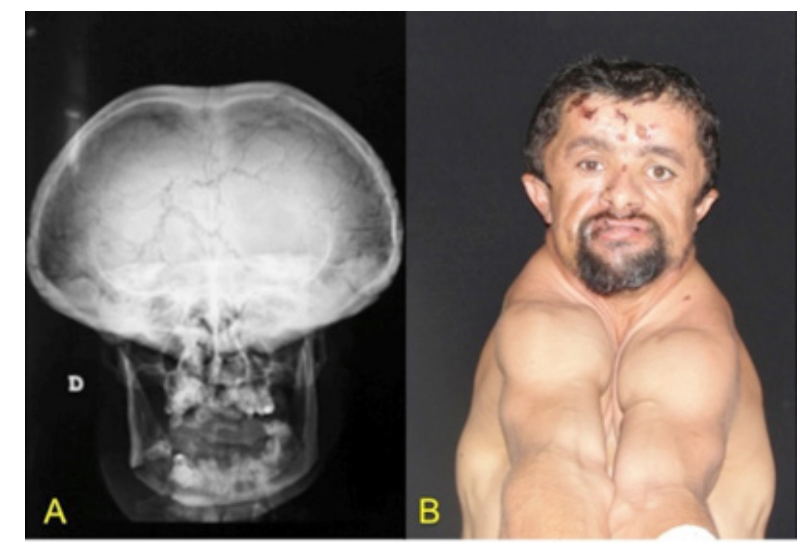

Figure 1: Presence of frontal and parietal projection observe the clavicular deformity.

In the postoperative period, the patient presented no pain, with good healing of the wounds and resolution of the edema and of the involved bruises. The patient was informed of the possibility of eruption of remaining impacted teeth and the possibility of using upper and lower dentures, but the same rejected the conduct. Three months after the surgery were performed imaging, when it was possible to observe adequate bone healing (Figure 1-4).

\section{Discussion}

Cleft cranial dysplasia is genetically mediated by the defect in the expression of the CBFA1 gene, which is responsible for the differentiation and osteoblastic formation, being essential for the formation of bone, both endochondral and intramembranous, being related to delayed ossification of the skull, pelvis, and on the CCD [5,6]. Molecular defects on chromosome 6p21 modify the translation of the RUNX2 binding gene, which is responsible for the induction of the CBFA1 gene in the differentiation of osteoblasts, bone formation, also participating in the process of odontogenesis $[2,5,6]$.

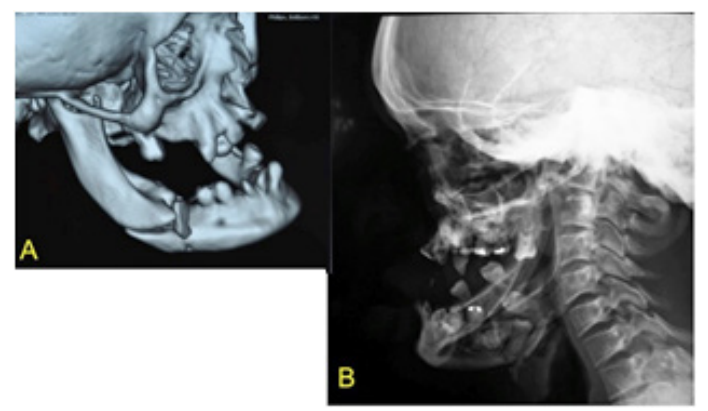

Figure 2: (a)Computed tomography showing comminuted fracture of the right mandibular angle. (b) Lateral oblique lateral of the right mandible showing the presence of impacted teeth at the fracture site.

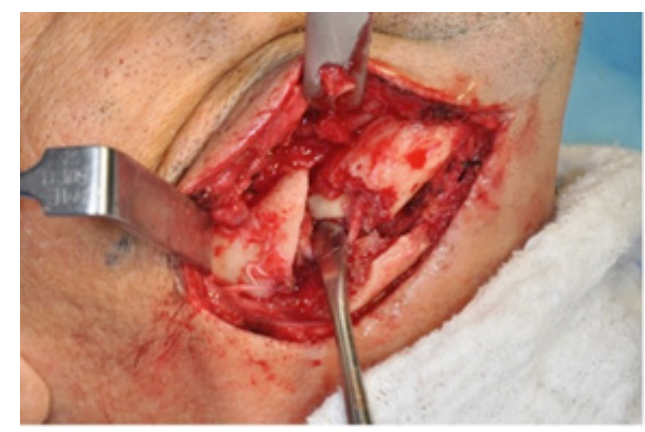

Figure 3: Fracture observed after submandibular access, arrow showing the presence of tooth and inferior alveolar nerve.

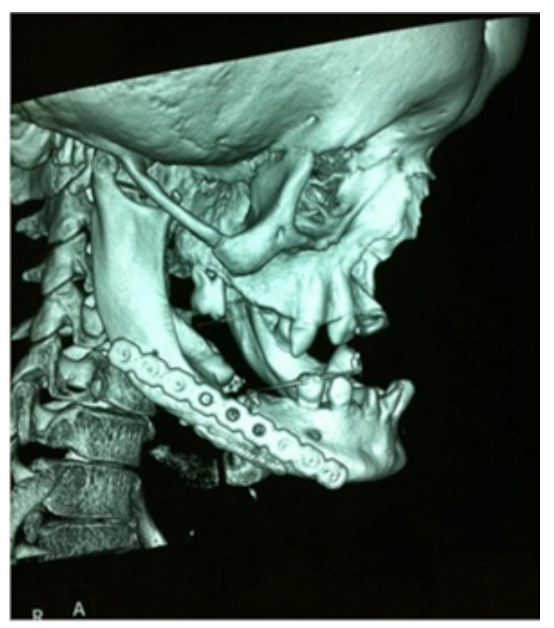

Figure 4: Fracture fixation with the use of $2.4 \mathrm{~mm}$, $2.0 \mathrm{~mm}$ plates and screws and screws postoperative control of the patient, three months after the surgical procedure. 
The diagnosis of cleidocranial dysplasia is based on clinical and radiographic signs of the patient. The professional should be attentive during the diagnosis, since some clinical features mimic cleidocranial dysplasia, and the presence of clavicular hypoplasia is a strong indication of this syndrome $[1,6,7]$. In the case reported, the patient presented impacted teeth, dental agenesis, hypoplasia of the clavicles and the middle face, ocular hypertelorism, enlargement of the frontal and occipital bones, and the presence of Wormian bones. In addition to the retention of deciduous teeth, many supernumerary teeth are present, especially in the mandible, which weakens the smaller amount of bone present [8], which was observed in the present case, where a low intensity trauma resulted in a fractured jaw in several segments.

The aesthetic and functional treatment of patients with dysplasia cleidocranial is multidisciplinary, involving the oral and maxillofacial surgery, orthodontics and prosthesis, in order to remove early supernumerary teeth, and the purpose of orthodontic stimulate permanent dentition or rehabilitate prostheses $[1,3,6]$.

In the proposed treatment of this patient, we observed that the case presented a satisfactory bone repair, with consolidation of all fracture sites and without any sign of infection. The large number of impacted teeth, given a lesser amount of bone tissue in fracture lines, which provided the greatest difficulties in surgical reduction and stabilization of the associated segments were fragmented segments of mandibular buccal and against which indicated the closed method. Was selected to open reduction and the use of 2.0 system boards to simplify the great fragmentation of fracture associated with it, we have chosen to use plates that supported the masticatory load without overloading the bone.

\section{Final Considerations}

The manifestations of cleidocranial dysplasia are of great interest to dentistry. Besides the difficulties in the area of prosthetic rehabilitation, the presence of multiple impacted teeth can cause serious problems such as the development of bone diseases and weakening of the mandible, as it happened in this case with a fractured mandibular angle.

\section{References}

1. Madeira MF, Caetano IM, Dias Ribeiro E, Rocha JF, Sonoda CK, et al. (2015) Orthognathic surgery in patients with cleidocranial dysplasia. J Craniofac Surg 26(3): 792-795.

2. Martins RB, De Souza RS, Giovani EM (2013) Cleidocranial dysplasia: report of six clinical cases. Spec Care Dentist 34(3): 144-150.

3. Noh K, Kwon KR, Ahn H, Paek J, Pae A (2014) Prosthetic rehabilitation of a cleidocranial dysplasia patient with vertical maxillofacial deficiency: a clinical report. J Prosthodont 23(1): 64-70.

4. Roberts T, Stephen L, Beighton P (2013) Cleidocranial dysplasia: a review of the dental, historical, and practical implications with an overview of the South African experience. Oral Surg Oral Med Oral Pathol Oral Radiol 115(1): 46-55.

5. Hardy A, Thiong'o MW, Leroy A, Hardy P, Laporte C (2015) Cleidocranial dysplasia with bilateral posterior glenohumeral dislocation: a case-report. Orthop Traumatol Surg Res 101(1):119-122.

6. Catunda I, Neto A, Landim F, Dourado I, Alcantara R, Leite E (2013) Manifestações maxilomandibulares de interesse odontológico nos pacientes portadores de displasia cleidocraniana. Relato de um caso clínico. Rev Cir Traumatol Buco maxilo facial Camaragibe 13(3): 25-30.

7. Feldman VB (2002) Cleidocranial dysplasia: a case report. 46(3): 185191.

8. Bharti K, Goswami M (2016) Cleidocranial dysplasia: a report of two cases with brief review. Intractable Rare Dis Res 5(2): 117-120. 